\title{
PLAN DE PREVENCIÓN DE ALERGIA AL LÁTEX EN EL ÁREA SANITARIA DE CIUDAD REAL
}

\author{
DRA.CARMEN MARROQUÍ LÓPEZ-CLAVERO*, \\ DRA.ISABEL SOLANO HERRERA**, DÑA Ma ISABEL RIESCO GARCÍA***
}

(*) Coordinadora Servicio de Prevención de Riesgos Laborales.

Médico Especialista en Medicina del Trabajo. Master en Prevención de Riesgos Laborales.

(**) Subdirectora Médico Hospital General de Ciudad Real. Jefe del Servicio de Prevención de Riesgos Laborales. Médico Especialista en Medicina del Trabajo. Master en Prevención de Riesgos Laborales.

(***) DUE de Empresa. Master en Prevención de Riesgos Laborales.

Master en Auditoría del Servicio de Prevención.

Servicio de Prevención de Riesgos Laborales Área Sanitaria de Ciudad Real.

Hospital General de Ciudad Real.

\section{RESUMEN}

Durante el año 2004 se instaura un Plan de Prevención de Alergia al Látex en el Área Sanitaria de Ciudad Real para cumplir con el Plan de Calidad del Servicio de Salud de Castilla la Mancha. Este plan se desarrollaría en tres vías de actuación y de forma simultanea, conteniendo medidas colectivas para intentar reducir el riesgo de sensibilización (formación e información de los trabajadores para el uso racional del guante y sustitución de los guantes de látex no estériles por vinilo o nitrilo), y medidas preventivas individuales tras la identificación, valoración y registro de los trabajadores alérgicos.

\section{PALABRAS CLAVES}

Látex, prevención, sensibilización, alergia, guantes, personal sanitario, registro.

\begin{abstract}
During the year 2004 a Plan of Prevention of Allergy is established to the Latex in the Sanitary Area of Ciudad Real to fulfill the Plan of Quality of the Service of Health of Castilla la Mancha. This plan would be developed in three performance roads and in a simultaneous way, containing collective measures to try to reduce the risk of sensitization (formation and the workers' information for the rational use of the glove and substitution of the non sterile latex gloves for vinyl or nitrilo), and individual preventive measures after the identification, valuation and the allergic workers' registration.
\end{abstract}

\section{KEY WORDS}

Latex, prevention, sensitization, allergy, gloves, sanitary personnel, registration. 


\section{OBJETIVO Y MÉTODO}

La sensibilización al látex es actualmente un problema importante, más de 40.000 productos tienen látex, y que puede tener graves consecuencias para la salud de la población llegando incluso a la muerte. Actualmente, aunque los datos son dispares, la prevalencia de alergia al látex en la población gene- ral, es de entre el $0.1 \%$ y el $2.3 \%$. Este porcentaje se eleva (3-12\%), en el caso de los trabajadores sanitarios. Es por ello, que instaurar un Programa de Prevención de la Sensibilización al Látex, ha sido y sigue siendo, uno de los objetivos marcados en el Plan de Calidad del Servicio de Salud de Castilla La Mancha (SESCAM).

\section{TRABAJADORES ESTUDIADOS}

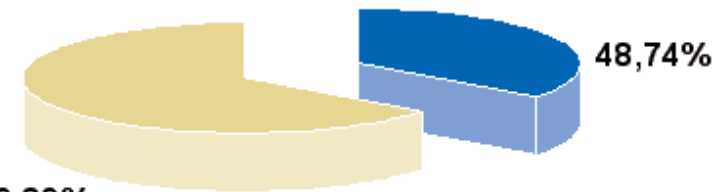

$89,29 \%$

\section{A.Especializada A.Primaria}

Figura 1. Respuesta de los trabajadores al estudio de alergia al látex.

Durante el año 2004, y para cumplir con este objetivo, el Servicio de Prevención de Riesgos Laborales, instauró un Plan de Prevención de Alergia al Látex en el Área Sanitaria de Ciudad Real. Este plan, contenía medidas preventivas colectivas para intentar reducir el riesgo de sensibilización (formación e información de los trabajadores para el uso racional del guante y sustitución de los guantes de látex no estériles por vinilo o nitrilo), y las bases para la creación de un procedimiento para identifi- car, valorar y registrar a los trabajadores alérgicos al látex, que nos permitiera la adopción de las medidas preventivas individuales que procedieran.

\section{DESARROLLO}

La primera acción a realizar en todo plan de prevención, es la difusión de su contenido. El plan, tenía tres puntos principales: La formación de los trabajadores para el uso racional del guante, la susti-

\section{UNIDADES ESTUDIADAS}

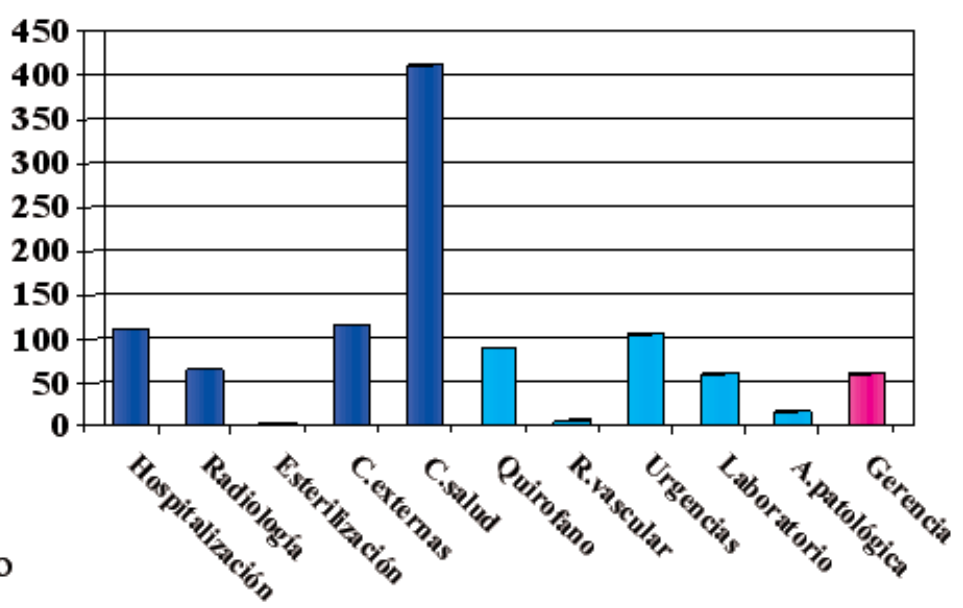

Alto

Figura 2. Unidades estudiadas de alto, moderado y bajo riesgo elegidas en A. Especializada y A. Primaria 


\section{TIPOS DE GUANTES HOSPITALARIOS DEL SESCAM}

\section{GUANTES ESTERILES}

GUANTES DE LÁTEX CIRUGIA CONPOLVO.

GUANTES DE LATTEX CIRUGIA SIN POLVO.

GUAMTES DE LATEX CURAS Y EXPLORACIONES SIN PQL WO

GUANTES NO LATEX MNEOPRENO)

GUANTES DE ATENUACION RADIOLOGICA

\section{GUANTES HOESTERILES}

GUAMTES DE VINILO

GUANTES DE NITRID

GUANTES DE POLIETL ENO (PLÁSTIOO)

\section{INDICACIONES GENERALES DE CADA TIPO DE GUANTES}

\section{GUANTES ESTERILES:}

\section{GUANTE QUIRURGICODE LÁTEX:}

\section{-ACTOS QUIRURGICOS.}

-SIEMAPRE QUE NO EXISTA ALERGIA A LÁTEX TANTO DEL PACIENTE COMO DEL PROFESIONAL SANITARIO.

\section{GUANTE QUIRURGICODE NEOPRENO.}

\section{-ACTOS QUIRÚRGICOS CUANDO EXISTA ALERGIA A} LÁTEX.

-EN PACIENTES EN PROTOCOLO SIN LÁTEX COMO

NEONATOS, NIÑOS CON MÁS DE TRES INTERVENCIONES, ESPINA BIFIDA, ATÓPICOS, ENFERMOS CON SONOAJES GENITOURINARIOS PROLONGADOS.

-CUANDO EXISTA ALERGIA A ACEL ERADORES QUIMICOS PRESENTES ENOTROS GUANTES.

\section{GUANTE DE CURAS DELÁTEX.}

-PROCEDIMIENTOS ESTERILES NO QUIRUROICOS YCURAS, CATETERISMO VESICAL, CATETERISMO VENOSO CENTRAL VIA PERIFERICA, CATETERISMO VENOSO

CENTRAL VAA SUBCLAVIA YO YUQULAR,

ADMINISTRACION NUTRICION PARENTERAL,...).

-SIEMPRE QUE NO EXISTA ALERGIA A LATEX TANTO DEL

PACIENTE COMODEL PROFESIONAL SANITARIO.

\section{GUANTES NO ESTERILES.}

GUANTE DE VINILO:

-PROCEDIMIENTOS NO ESTERILES.

-CATETERISMO VENOSO VIA PERIFÉRICA

-EXTRACCIONES SANGUINEAS, ADMINISTRACION DE MEDICACION, EXCEPTO CITOSTATICOS,..)

-MANIPULACION DE MUESTRAS SIEMPRE QUE ESTÉN CONTENIDASEN TUBO CERRADO.

-ASEO E HIGIENE DEL PACIENTE.

-LIMPIEZA DE MATERIAL SANITARIO.

GUANTE DE NITRILO:

-REALIZACIONDE LAS DIFERENTES TECNICAS EN LOS LABORATORIOS (ANALISIS CLÍNICOS, MICROBIOLOGIA, HEMATOLOGIA, ANATOMIA PATOLÓGICA...)

-MANIPULACION DE PRODUCTOS QUIMICOS Y CITOTOXICOS (PREPARACION Y ADMINISTRACIÓN).

-ASEO E HIGIENE DE PACIENTES AISLADOS (MANEJO DE EXCRETAS, )

-ASEO E HIGIENE DE PACIENTE EN TTO CON CITOTÓXICOS (MANEJO DE EXCRETAS).

-ASEO E HIGIENE DE PACIENTE QUE HAN RECIEIDO RADIOFÁRMACOS (MANEJO DE EXCRETAS).

GUANTE DE POLIETILENO (PLÁSTICO): -PROCEDIMIENTOS NO ESTERILES DE BAJO RIESGO.

\section{RECOMENDACIONES GENERALES DEL USO DE GUANTES}

-EL GUALTE SANITARIO ES UNAA BARRERA BIDIRECCIONAL ENTRE PACIDNTE Y PROFESIONAL SAKITARIO.

- LOS GUANTES DEBEN SER CAMIBLAD OS ENTRE CADA PACIENTE Y ENTRE CADA PROCEDMUIENTO

-EI GUANTE DEBESER EI ADECUADO PARA CADAPROCED IMIENTO -LAVAR LAS MAANOS AHTES Y DESPUES DE CADA USO DE GUANTES. -HO USAR CREMLAS DE MASOSOS ANTES DE USAR GUANTES YA QUE ROMPEMOS EL EFECTO BARRERA DEL GUANTE.

-EI GUANTE DEBESER EI ADECUADO PARA CADAPACIENTE Y SU SITUACION ESPECIFICA (ALERGLA A. LASTEX).

- CONSIDERAR SITUACIOHES ESPECIFICAS DEI TRABAJADOR SANITARIO AHTES DE ELEFIR EI GUANTE (TRABAJADORES ALERGICOS A LATEX).

- CAMELLR EL GUAFTE DURALTE SU USO SIFMTPE QUESE HAYA ROTO CONTAMINADO

\section{CONSIDERACIONES GENERAIES}

-DEB EMTOS PREVENIR LA SENSIRIIZACION Y REACCIONES ALERGICAS AL LATEXX EN EI AMBITO SANITARIO.

-AL ESCOGER EL GUANTE DEBEMTOS TENER EN CUENTA: -SITUACIONES ESPECLALES DEL PACIENTTE. -STTUACIONTS RSPECIAIAS DET TRABAJADOR. -PROCED IYIEVTTO P ARA EI QUESE VA A UTILIRAR. - CAMIBLAR DE GUAFTES AL FIHALIZAR EI PROCEDIMIENTO Y ENTRE P ACIENTES

CUAND 0 EXTSTAN DUDAS EN QUE TIPO DE GUANTE UTILIZAR EN UN

DETRRMNADOPROCEDTMIFNTO CONSULTAD AL SPRL 0 A

SUP ER UISORA DE ENFERIERIA DE LA UNID AD 


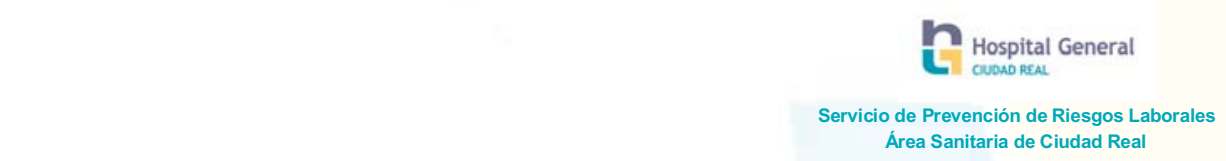

CUESTIONARIO ALERGIA A LÁTEX

\begin{tabular}{|c|c|c|c|}
\hline • & ¿Se agrietan sus manos cuando se pone guantes de látex? & $\square$ Sí & $\square$ No \\
\hline • & $\begin{array}{l}\text { ¿Durante o después de su jornada laboral nota que le pican o se le hinchan los ojos o } \\
\text { padece aumento de secreción nasal? }\end{array}$ & $\square$ Sí & $\square$ No \\
\hline • & ¿Padece múltiples alergias a polen, ácaros, metales...? & $\square$ Sí & $\square$ No \\
\hline • & $\begin{array}{l}\text { ¿Sufre ataques de asma o utiliza el inhalador con más frecuencia en el lugar de } \\
\text { trabajo? }\end{array}$ & $\square$ Sí & $\square$ No \\
\hline • & ¿Jadea o sus labios y cara se hinchan cuando infla un globo? & $\square$ Sí & $\square$ No \\
\hline - & $\begin{array}{l}\text { ¿Tiene irritación vaginal, picor, escozor tras examen ginecologico o relaciones } \\
\text { sexuales con uso de condón? }\end{array}$ & $\square$ Sí & $\square$ No \\
\hline - & ¿Ha sufrido múltiples intervenciones quirúrgicas? & $\square$ Sí & $\square$ No \\
\hline • & $\begin{array}{l}\text { ¿Tiene alguna alergia alimentaria?. La alergia al látex se asocia también con la alergia } \\
\text { a algunos alimentos, en particular a los aguacates, patatas, plátanos, tomates, } \\
\text { castañas, kiwis y papaya. }\end{array}$ & $\square$ Sí & $\square$ No \\
\hline • & ¿Padece de espina bífida? & $\square$ Sí & $\square$ No \\
\hline • & ¿Padece anomalías congénitas del tracto urinario? & $\square$ Sí & $\square$ No \\
\hline
\end{tabular}

Tabla II. Cuestionario de Despistaje de Posibles Sensibilizaciones al látex

PATOLOGÍA REFERIDA

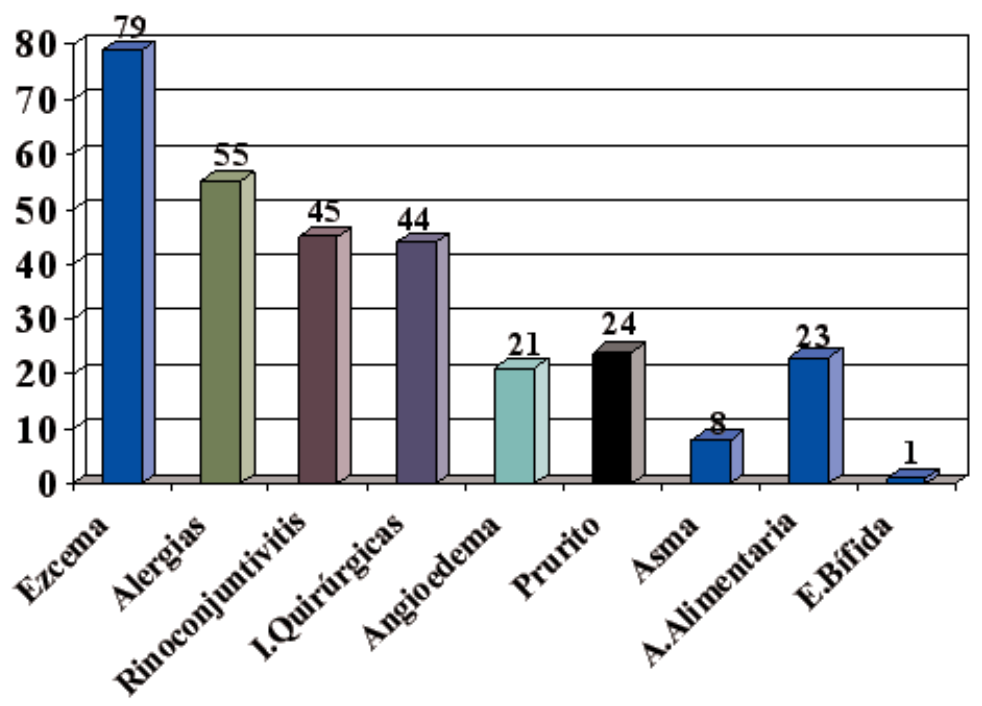

Figura 3. Sintomatología en trabajadores sospechosos de alergia al látex. 


\section{PATOLOGÍA PREDOMINANTE EN SENSIBILIZADOS}

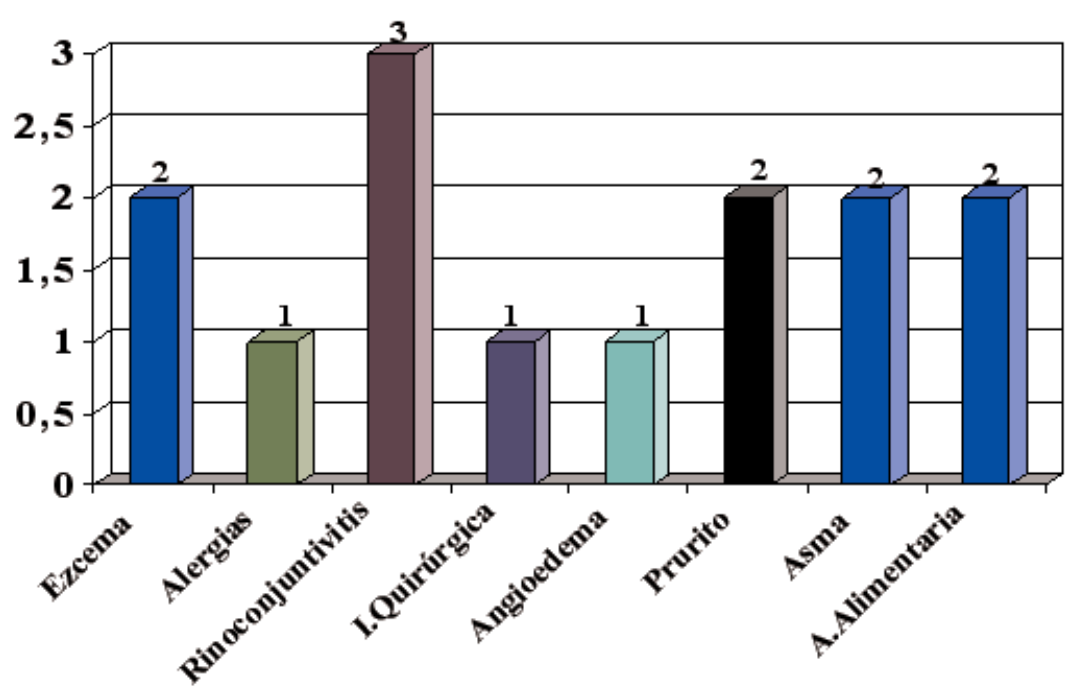

Figura 4. Sintomatología en trabajadores alérgicos al látex.

tución de los guantes de látex no estériles en toda el área y la creación de un registro de alérgicos al látex. Explicamos el contenido del plan, tanto a las Gerencias del Área Sanitaria de Ciudad Real (Especializa- da y Primaria), como a los trabajadores y sus representantes, pidiendo su colaboración. Conseguida la autorización e implicación en el proyecto de ambas Gerencias, procedimos a planificar las actuaciones.

\footnotetext{
Hospital General
Servicio de Prevención de Riesgos Laborales
Área Sanitaria de Ciudad Real
}

sescam 8

\section{REGISTRO DE TRABAJADORES ALÉRGICOS AL LÁTEX}

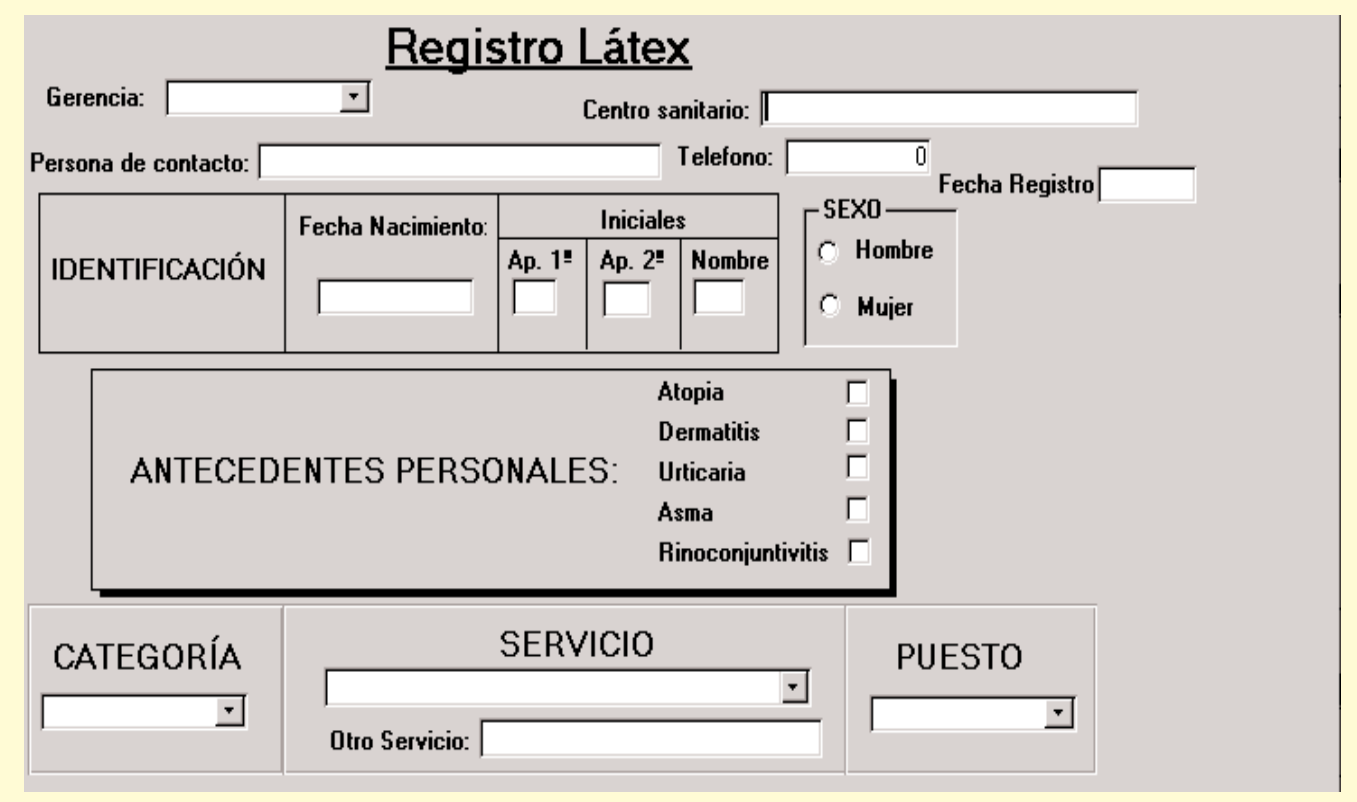

Tabla III. Datos personales y laborales del trabajador alérgico al látex. 


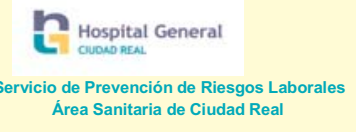

\section{REGISTRO DE TRABAJADORES ALÉRGICOS AL LÁTEX}

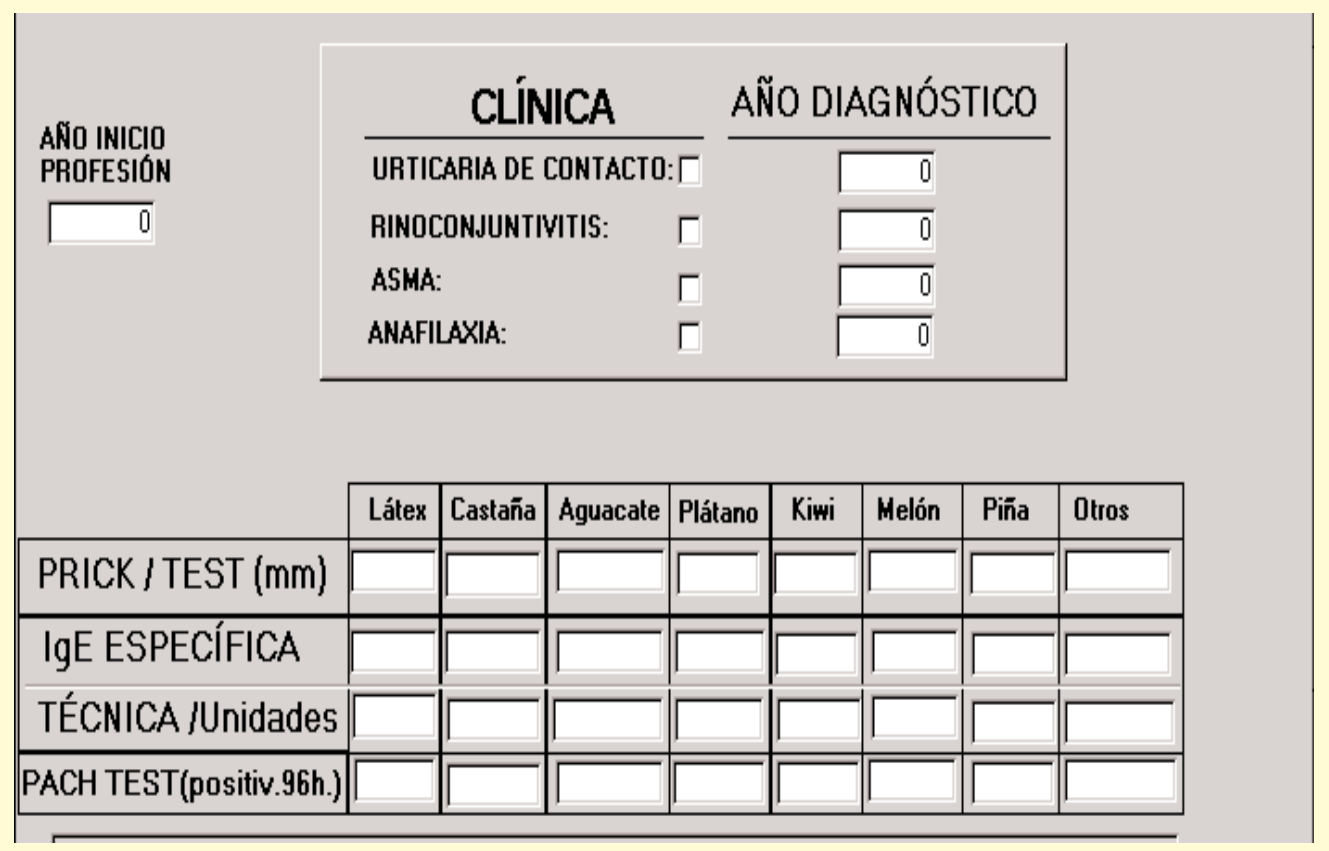

Tabla IV. Clínica y pruebas especificas del trabajador alérgico al látex.

La primera decisión importante que tomamos, fue que el inicio y desarrollo de las tres vías de actuación, iba a ser de forma simultánea y no en fases.

Las acciones realizadas en cada trabajadores sanitarios vía, fueron las siguientes:

\section{FORMACIÓN DE LOS TRABAJADORES}

Se envío a las Direcciones de Enfermería y de Gestión de Atención Especializada y de Primaria del Área Sanitaria de Ciudad Real póster informativo que contenían los tipos de guantes hospitala-

\section{PREVALENCIA ALERGIA AL LÁTEX ÁREA CIUDAD REAL}

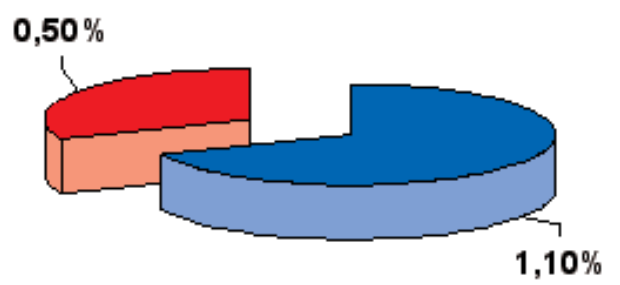

A.Especializada

$\square$ A.Prim aria

Figura 5. Prevalencia de trabajadores alérgicos en A. Especializada y A. Primaria. 
rios del sescam, las indicaciones generales de cada tipo de guante y las recomendaciones de uso de guantes. Los posters fueron distribuidos por servicios centrales, plantas de hospitalización, consultas externas y centros de salud. Asimismo, también se realizaron charlas informativas sobre el uso racional del guante.

\section{SUSTITUCIÓN DE GUANTES DE LÁTEX NO ESTÉRILES}

Realizamos un análisis exhaustivo del consumo de guantes desde el año 2001 al 2003 por servicios, por materiales y por tipo de guante, valorando en todos los casos la ecuación coste / eficacia / beneficio, tanto para el paciente como para el trabajador.

\section{REGISTRO DE TRABAJADORES SANITARIOS ALÉRGICOS AL LÁTEX}

Para la elaboración del registro, lo primero que hicimos fue clasificar las unidades asistenciales en unidades de alto, moderado y bajo riesgo de sensibi- lización al látex. Con esta actuación lo que buscábamos, era estudiar unidades de alto, moderado y bajo riesgo de forma aleatoria y conjunta, para que así, los datos de prevalencia de alergia al látex en nuestro hospital que fuéramos obteniendo no se desviaran demasiado y nos permitieran ir tomando medidas preventivas. El criterio utilizado para esta clasificación fue el consumo de guantes.

Los pasos seguidos para la elaboración del registro fueron:

1. Envío a las distintas unidades de alto, moderado y bajo riesgo elegidas, y para ser cumplimentado por los trabajadores, de un cuestionario para el despistaje de posibles sensibilizaciones al látex.

2. Valoración en el Servicio de Prevención de los cuestionarios, citando a los trabajadores que fueran sospechosos de alergia al látex para la realización de un segundo cuestionario más específico.

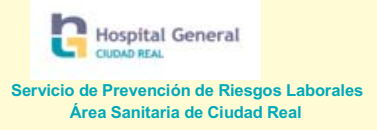

\section{REGISTRO DE TRABAJADORES ALÉRGICOS AL LÁTEX}

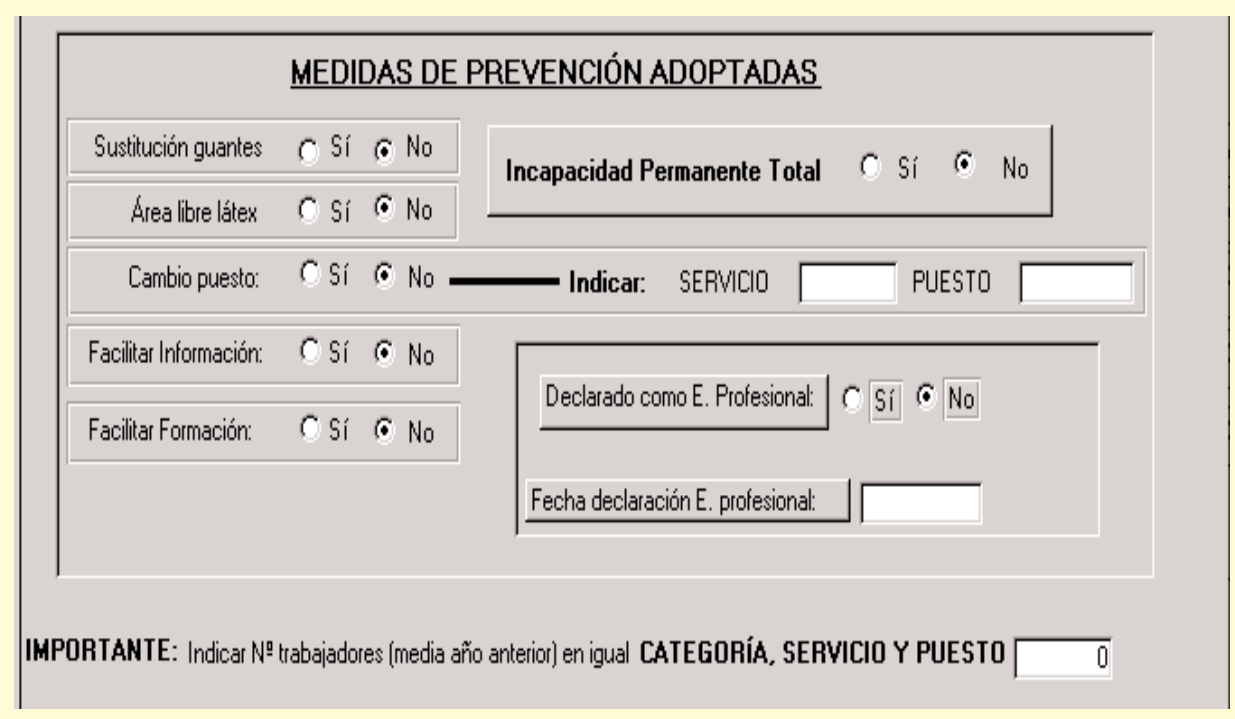

Tabla V. Adopción de medidas preventivas individuales. 
3. Apertura de historia clínica a aquellos trabajadores que tras el segundo cuestionario siguieran siendo sospechosos de alergia al látex.

4. Cita para la realización de pruebas específicas (Prick Test, IgE específica CAP y Pach Test) para el diagnóstico definitivo de certeza.

5. Elaboración del Registro electrónico de trabajadores alérgicos al látex, incluyendo: Gerencia a la que pertenece, datos personales, puesto de trabajo, clínica de alergia al látex, pruebas especificas realizadas y medidas preventivas adoptadas.

\section{RESULTADOS}

La aceptación y participación de los trabajadores en las actividades de formación fue todo un éxito: Se solicitaron más ejemplares de los posters informativos, y se realizaron constantes consultas al Servicio de Prevención sobre los guantes que se debían utilizar en las distintas actividades.

En el estudio realizado en el año 2004 del consumo de guantes de látex no estériles, detectamos que entre los años 2001-2003 se había producido un aumento del $27,31 \%$ en el consumo total de guantes de látex no estériles en el Hospital General de Ciudad Real, en detrimento de los guantes de vinilo. Esto suponía, que durante ese periodo, se había producido un aumento del riesgo de sensibilización al látex de los trabajadores. Para disminuirlo, se procedió a la sustitución del guante de látex no estéril por vinilo o nitrilo y a su eliminación del pacto.

En cuanto al Registro de Trabajadores Alérgicos al Látex, en el estudio realizado en el periodo que va desde junio de 2004 a mayo de 2006, la respuesta fue muy desigual en Atención Especializada y en Atención Primaria. Mientras en Atención Especializada sólo respondieron el 48,74\% de los trabajado- res encuestados (271 cuestionarios de los 556 enviados) llegando incluso a ser menor en unidades de alto riesgo como los quirófanos (24,44\%), en Atención Primaria la respuesta fue del 89,29\% (367 cuestionarios de los 411 enviados).

La prevalencia de trabajadores alérgicos al látex en las unidades estudiadas, fue del $1,10 \%$ en Atención Especializada y del 0,5\% en Atención Primaria, dato este último explicable, al no haber estudiado ninguna unidad catalogada como de alto riesgo en Atención Primaria.

La categoría profesional con mayor sensibilización fueron las DUE, seguidas por las Auxiliares de Enfermería, con una experiencia profesional mayor de 30 años y sintomatología preferente de rinoconjuntivitis seguida de asma, irritación y eczema.

\section{CONCLUSIONES}

La aceptación del Plan de Prevención de Alergia al Látex, tanto de las Gerencias de Especializada y Primaria del Área Sanitaria de Ciudad Real, como de los trabajadores y sus representantes sindicales, ha sido muy satisfactoria.

Los logros obtenidos gracias al plan son la desaparición prácticamente del guante de látex ambidiestro no estéril, el de más amplia difusión hospitalaria y responsable en gran medida de la sensibilización al látex por su alto contenido en proteínas y su vehiculación en el polvo, siendo sustituido dentro de los servicios por nitrilo y vinilo según el uso, la formación de los trabajadores en el uso racional del guante del vinilo y nitrilo según el tipo de actividad en los distintos servicios, la realización de un catálogo de guantes a utilizar en el Hospital General de Ciudad Real, y la creación de un registro de trabajadores sanitarios alérgicos al látex, que ha permitido tomar medidas preventivas individuales. 


\section{BIBLIOGRAFÍA}

1. Plan de Calidad SESCAM 2006.

2. Blanco C, Quirce. S.Alergia al látex. Mra ediciones; 2002.

3. Unidad de Salud laboral Comarca de Bilbao. Adecuacion de Consulta ATS-EAP, consulta médico EAP, sala de curas y sala de extracciones por alergia al látex. Enero 2001.

4. Vandenplas O, Delwiche JP, EVard G, Aimont P, van der Brempt X, Jamart J, et al. Prevalence of occupational asthma due to latex among hospital personnel. Am J Respir Crit care Med 1995; 151:54-60.

5. Sussman GL, Liss GM, Deal K el al. Incidence of latex sensitisation among latex glove users. J Allergy Clin Immunol 1998; 101; 171-178.
6. Swanson MC, Bubak ME, Hunt L, Yunginger JW, Warner MA, Reed CE, Quantification of occupational latex aeroallergens in a medical center. J Allergy Clin Immunol 1994; 94:445-451.

7. Allmers H, Brehler R, Chen Z, Raulf-Heimsoth M, Fels H, Baur X. J Reduction of latex aeroallergens and latex-specific IgE antibodies in sensitized workers after removal of powdered natural rubber latex gloves in a hospital. Allergy Clin Immunol. 1998 Nov; 102(5): 841-6.

8. Mahler V, Fisher S, Fuchs T, Ghannadan M, Valent P, Fartasch M, et al. Prevention of latex allergy by selection of gloves. Clin Exp Allergy 2000; 30:509-520. 
